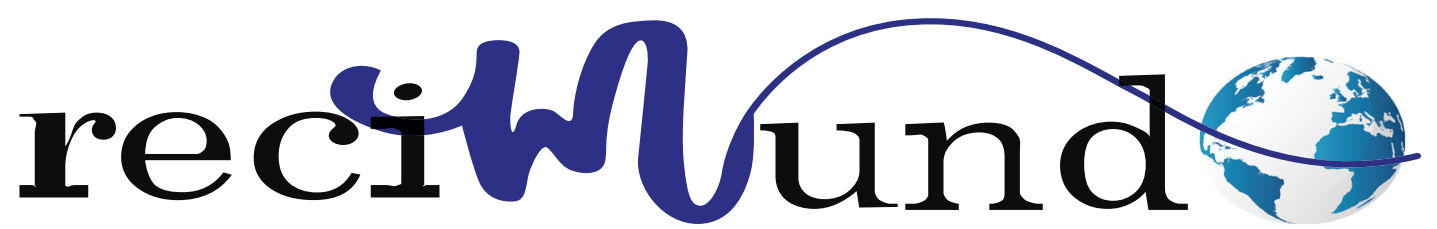

Revista Científica Mundo de la Investigación y el Conocimiento

DOI: 10.26820/recimundo/5.(Suple1).oct.2021.44-55

URL: https://recimundo.com/index.php/es/article/view/1331

EDITORIAL: Saberes del Conocimiento; Universidad Yachay Tech

REVISTA: RECIMUNDO

ISSN: 2588-073X

TIPO DE INVESTIGACIÓN: Artículo de revisión

CÓDIGO UNESCO: 5312.90 Turismo

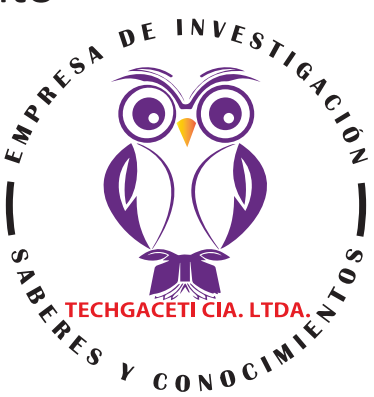

PAGINAS: 44-55

\title{
El turismo en salud y atención primaria ambiental. Caso: complejo turístico Shuar Tsuer Entsa-Balao Chico Ecuador
}

Tourism in health and primary environmental care. Case: Shuar Tsuer Entsa-Balao Chico tourist complex Ecuador

Turismo em saúde e cuidados ambientais primários. Caso: Complexo turístico Shuar Tsuer Entsa-Balao Chico Equador

Cruz Xiomara Peraza de Aparicio ${ }^{1}$

RECIBIDO: 02/09/2021 ACEPTADO: 20/09/2021 PUBLICADO: 30/10/2021

1. Especialista en Medicina General de Familia; Doctora en Ciencias de la Educación; Magister en Educación Mención Planificación Educativa; Médico Cirujano; Carrera Enfermería; Universidad Metropolitana del Ecuador; Guayaquil, Ecuador; cperaza@umet.edu.ec; iD https://orcid.org/0000-0003-2588-970X

CORRESPONDENCIA

Cruz Xiomara Peraza de Aparicio

cperaza@umet.edu.ec

Guayaquil, Ecuador

( RECIMUNDO; Editorial Saberes del Conocimiento, 2021 


\section{RESUMEN}

Ecuador, es un país con una rica biodiversidad, muy propicia para el ejercicio del turismo, cuenta con una extensa variedad de culturas, y un extraordinario patrimonio histórico. Está incluido entre los 14 países megadiversos del mundo, con el honorable título de ser uno de los países mejor preservados ambientalmente. Objetivo: valorar la relación entre las actividades turísticas en el Complejo Turístico Shuar Tsuer Entsa y la conservación del medio ambiente. Material y métodos: Se realizó una investigación cualitativa, levantando un cuadro de doble entrada para evaluación del impacto ambiental. Resultados: La estrecha relación que existe entre conservación del medio ambiente receptor y el crecimiento de la actividad turística, plantea una interrogante para la gestión de las autoridades regionales y locales, así como para los habitantes de ese espacio geográfico natural, referente a los impactos ambientales que circundan al Complejo turístico y la manera de compatibilizar el desarrollo turístico desde la Atención Primaria Ambiental. Conclusión: se pretende con esta visión basada en los resultados el logro de un desarrollo humano con calidad de vida, con una visión de comunidades participativas, y una atención de salud democrática, verdaderamente humanística, para la prestación de un servicio turístico acorde con la filosofía del Buen Vivir.

Palabras clave: Termalismo, Turismo en Salud, impacto ambiental.

\section{ABSTRACT}

Ecuador is a country with a rich biodiversity, very conducive to the exercise of tourism, has a wide variety of cultures, and an extraordinary historical heritage. It is included among the 14 megadiverse countries in the world, with the honorable title of being one of the best environmentally preserved countries. Objective: assess the relationship between tourist activities in the Shuar Tsuer Entsa Tourist Complex and the conservation of the environment Material and methods: A qualitative research was carried out, creating a double entry table for environmental impact assessment. Results: The close relationship that exists between conservation of the receiving environment and the growth of tourist activity raises a question for the management of regional and local authorities, as well as for the inhabitants of this natural geographic space, regarding environmental impacts. that surround the Tourist Complex and the way to reconcile the tourist development from the Primary Environmental Care. Conclusion: this results-based vision is intended to achieve human development with quality of life, with a vision of participatory communities, and truly humanistic, democratic health care for the provision of a tourist service in accordance with the philosophy of Good Living.

Keywords: Key Words: Thermalism, Health Tourism, environmental impact.

\section{RESUMO}

O Equador é um país com uma rica biodiversidade, muito propício ao exercício do turismo, possui uma grande variedade de culturas e um extraordinário patrimônio histórico. Está entre os 14 países megadiversos do mundo, com o honroso título de ser um dos países mais bem preservados do meio ambiente. Objetivo: avaliar a relação entre as atividades turísticas no Complexo Turístico Shuar Tsuer Entsa e a conservação do meio ambiente. Material e métodos: Foi realizada uma pesquisa qualitativa, criando uma tabela de dupla entrada para avaliação de impacto ambiental. Resultados: A estreita relação que existe entre a conservação do meio receptor e o crescimento da atividade turística levanta uma questão para a gestão das autoridades regionais e locais, bem como para os habitantes deste espaço geográfico natural, no que se refere aos impactos ambientais. que circundam o Complexo Turístico e a forma de conciliar o desenvolvimento turístico com a Atenção Básica Ambiental. Conclusão: esta visão baseada em resultados pretende alcançar o desenvolvimento humano com qualidade de vida, com uma visão de comunidades participativas, e de saúde verdadeiramente humanística e democrática para a prestação de um serviço turístico de acordo com a filosofia do Bem Viver.

Keywords: Key Words: Thermalism, Health Tourism, environmental impact 


\section{Introducción}

A partir de la consolidación de lo que se ha denominado la Nueva Era del Turismo (NET), donde predomina la motivación por participar de experiencias de ocio y recreación vinculadas al tema ambiental, y cuando la calidad constituye un factor determinante en la valoración que se le asigna a los productos turísticos, puede afirmarse que el objetivo de alcanzar un desarrollo sostenible no sólo tendrá un efecto positivo en el ámbito ecológico y socio cultural, sino que en muchas zonas, y principalmente en el entorno rural, será una condición básica para asegurar la presencia del turismo como actividad económica (Fayos Solá, 1997; Ministerio de Comercio y Turismo de España, 1994).(Loor, Alonso y Pérez; 2017)

Como integrante del turismo de salud, se encuentra el termalismo. A nivel mundial durante la última década ha experimentado un gran dinamismo y crecimiento. Alrededor del siglo XX, explica Rivas (2020), nace el consumo masivo y a la par se revaloriza el tiempo libre de las personas, se incrementa la búsqueda de tratamientos y procedimientos médicos fuera de fronteras nacionales por diferentes razones ha creado una oferta internacional de servicios de salud, que combinan los procedimientos de medicina curativa, preventiva, estética y de bienestar con experiencias turísticas basadas en riquezas naturales, culturales, artesanales y autóctonas de cada país oferente.

En América Latina, refiere Suarez (2016), que se ha incrementado un 35 por ciento el número de personas interesadas en pasar sus vacaciones en estaciones termales, centros de talasoterapia o SPA, mientras que los ingresos económicos en reservas han aumentado cerca de un 40 por ciento, respecto al año 2000.

Al considerar el turismo, en su esencia, como una actividad compleja que involucra relaciones humanas y socio-espaciales, además de variados sectores económicoproductivos, Wallingre (2018) se propone dar respuesta a algunos interrogantes que intervienen en su desarrollo, a partir de delimitar su abordaje desde la denominada etapa del turismo industrial maduro (que se inicia estimativamente en 1945) y hasta la actualidad, mediante los casos de Argentina, Brasil, Chile, Cuba, México, Perú y Uruguay En definitiva, el termalismo del nuevo milenio demanda mayor dinamismo y operatividad, evolucionar sin renunciar a la parte científica que aportan las aguas termales a la salud.

El Ecuador, es un país con una rica biodiversidad, muy propicia para el ejercicio del turismo, se presentan externalidades que han acelerado el deterioro de los ecosistemas y ha contribuido a profundizar la pobreza en áreas rurales deprimidas, y que en la actualidad se encuentran en posición de conflicto por múltiples razones de diversa índole, ya sea por la minería, la producción industrial o la explotación forestal Estas formas de intervención generan impactos ambientales, que en el corto plazo reducen su capacidad de proveer bienes y servicios derivados de la producción de recursos naturales, y su capacidad de atraer nuevos visitantes y residentes a las áreas rurales.

Esta situación constituye una de las principales limitantes para alcanzar las expectativas de crecimiento turístico planteadas por el sector privado, así como para el éxito de las estrategias gubernamentales que le asignan una importancia significativa al agroturismo y ecoturismo como actividades prioritarias para algunas áreas donde otras actividades productivas presentan limitaciones (Brandon, 1993; Fuentes, 1995; Rivas, 1996; Martínez, 1998).

Por tratarse de un tema de salud e impacto ambiental, se toma como pilar primordial lo expresado por la Organización Panamericana de la Salud (OPS; 1997), la cual expresa que la atención primaria ambiental es una 
estrategia de la acción del medio ambiente, básicamente preventiva y participativa, que reconoce el derecho del ser humano a vivir en un ambiente saludable y agradable y a informarse acerca de los riegos ambientales sobre la salud, el bienestar y la supervivencia.

En cuanto al termalismo del Complejo Turístico Shuar Tsuer Entsa ubicado en el cantón Naranjal, el objetivo del estudio es valorar la relación entre las actividades turísticas en el Complejo Turístico Shuar Tsuer Entsa y la conservación del medio ambiente, la metodología empleada para la Evaluación del Impacto Ambiental (EIA) es el Método de Leopold, considerando los factores externos; que afectan para la consolidación del termalismo en el complejo de una manera más ecológica, considerando los principios de la Atención Primaria del medio ambiente. Esta matriz de Leopold según Gómez (2019), sistematiza la relación entre las acciones a implementar en la ejecución de un proyecto y su posible efecto en factores ambientales.

\section{Desarrollo}

En términos generales el turismo a nivel mundial ha experimentado un crecimiento importante según cifras estadísticas de la UNWTO Tourism Highlights (2015), esta industria que ha pasado de tener 25 millones de turistas en 1950 a más de 1.100 millones en 2014,encontrándose dentro de su clasificación, el denominado turismo de salud, cuya definición internacional recibe distintos modos de interpretación, generalmente se asocia con todas las actividades orientadas al uso de aguas mineromedicinales para preservar la salud. Silega (2010, pg. 14), indica que este es "el proceso en el cual una persona viaja por fuera de su lugar de residencia con el objetivo primordial de recibir servicios de salud o de bienestar".

Los turistas en viajes de salud son denominados Curistas y son tratados en centros balneoclimatológicos y Kurthoteles. Según Correa (2017), América Latina se ha posicionado como líder, destacando países como Brasil, México y Colombia que cuentan con una oferta caracterizada por costos bajos junto con un portafolio diversificado de especialistas de alto nivel internacionalmente reconocido y centros de calidad diseñados para garantizar una experiencia de calidad en salud. El contacto directo con la naturaleza permite relacionarse más estrechamente con la topografía del país y aprovechar estas bondades naturales para sanar afecciones tanto físicas como psicoespirituales producto del estrés que se vive en las ciudades.

Este tipo de turismo, refiere Silega (2010, pg14) puede clasificarse como Turismo asistencial de salud, donde el individuo busca técnicas científicas avanzadas en diagnósticos y tratamientos a un costo inferior al de su país de origen. El otro corresponde al Turismo natural de salud donde la búsqueda del individuo se centra en la utilización de factores naturales (sol, agua, mar), fuera de su lugar de procedencia, en ambos casos pretende obtener un completo bienestar físico, mental y social. Otra clasificación del turismo en salud explica Secall (2006, pg174) diferencia el segmento en: turismo termal o de balneario, el clínico, el de belleza y el geriátrico.

Es importante la revisión que plantea Mourelle (2009), en cuanto a la clasificación de los centros según el tipo de agua con fines terapéuticos y de bienestar: En primer lugar, están los balnearios y estaciones termales: Centros sanitarios que usan aguas mineromedicinales con fines preventivos y terapéuticos. En segundo lugar, están los centros de Talasoterapia, aquellos espacios que utilizan el agua de mar con fines preventivos y terapéuticos, finalmente los Centro Spa, que son centros no sanitarios que aplican técnicas hidrotermales y estéticas, usando agua común, con o sin aditivos. 
En América Latina, refiere Rivas (2020), las aguas termales son un destino bastante escogido como opción turística por muchas personas, que cada vez más confían en sus beneficios y promueven unas vacaciones saludables. En opinión de Santos (2016), Argentina es un país con gran potencial de desarrollo del turismo de bienestar y del termalismo en la región, el turista doméstico busca programas de estilo de vida saludable, anti ageing y longevity, agregando como dato que "el consumidor de productos wellness lo demanda también en sus viajes" y que la particularidad de los internacionales es que buscan experiencias, razón por la que recomendó a los destinos hacer una "apuesta por la singularización de los tratamientos".

Según el Ministerio de Turismo(2012,pg 6) el Ecuador es un destino turístico que ofrece varias alternativas de actividades para el viajero: desde el turismo de aventura al ecoturismo, aviturismo, agroturismo, turismo en salud, voluntariado, historia y cultura, deportes acuáticos, viajes de incentivos y convenciones, siendo importante significar que el país posee cuatro regiones que van desde la diversidad topográfica de la Amazonía a volcanes y nevados de los Andes; amplias playas en la Costa y las tan conocidas Islas Galápagos, conocidos como las islas encantadas, en la región insular.

Cada una de las cuatro representa un ecosistema único y por lo tanto es el país que cuenta con la mayor biodiversidad por kilómetro cuadrado, debido a su amplia diversidad y ubicación geográfica, se ha declarado Patrimonio Natural de la Humanidad por la UNESCO (1978).

En cuanto a las prácticas de termalismo en el Ecuador, estas se realizan según Calderón (2016), en unos 85 afloramientos termales con un flujo que los hace utilizables para el bienestar humano, muchos tienen accesibilidad vial, varios de ellos han sido adecuados en condiciones muy similares a las de un balneario de agua común, que privilegia lo lúdico.

En la corriente conservadora existe una noción muy generalizada de sanación de las aguas termales de los dolores musculares, por lo que es común encontrar en los baños termales a gente de la tercera edad o gente joven que busca recuperarse de algún tipo de accidente que puede ser desde una torcedura de tobillo hasta sobrevivir a un grave accidente de tránsito.

El termalismo, es un atractivo inigualable que convierte a las ciudades que tienen la fortuna de poseer aguas termales en destinos obligados de convocatoria local o mundial, dependiendo de la calidad de sus instalaciones, si bien las aguas termales son muy similares en sus propiedades curativas, relajantes, antiestrés, mucho depende, de sus municipios el buen uso y rentabilidad que den las mismas a la ciudad y sus ciudadanos.

Se resalta según Toledo \& Aparicio (2018), que en la provincia de Guayas se encuentra el Centro Shuar de Aguas Termales, ubicado a $8 \mathrm{Km}$. de la vía Panamericana, pasando el cantón Naranjal, a la entrada de Balao Chico, a una hora y media de Guayaquil, aquí convergen una diversidad de animales exóticos, entre ellos: guantas, conejos, loros, diversos tipos de monos; también hay una gran diversidad de anfibios e insectos. La flora en el sector permite apreciar chisparo, matapalo, cade, clavellin, laurel, guaba de río, entre otros. Existen plantas de roca como la toquilla de roca, begonias silvestres, helechos epifitos, caña guadua, entre otros.

El Centro Turístico consta de dos piscinas: La primera tiene 1 metro de profundidad y una temperatura de $40^{\circ}$. La segunda tiene 1,50 metros de profundidad y una temperatura es de $40^{\circ}$. En el sitio cuenta con vestidores y varios chorros de agua fría y caliente. (Toledo \& Aparicio,2018) 
En cuanto a la Atención Primaria Ambiental (APA), esta surge de la fusión de dos vertientes, una la estrategia de Atención Primaria en Salud, cuyo propósito fue lograr salud para todos en el año 2000 y la otra surge del Desarrollo Rural Integrado, el cual constituyó un marco de políticas agrarias para los países del tercer mundo en la década de los años setenta. Este último modelo permitió virar el paradigma de desarrollo centrado en elementos de cantidad y productividad por el de calidad de vida de las poblaciones marginales y cuyo concepto de pobreza se centraba en la capacidad adquisitiva de bienes y servicios.

Retomando el propósito de la Atención Primaria en Salud, ésta conceptualizó en su origen el desarrollo humano con calidad de vida, con una visión de comunidades participativas, y una atención de salud democrática, verdaderamente humanística. A la vez, considera los aspectos técnicos de sus programas como insumos importantes y complementarios, pero no lo esencial del mismo. Para ello propone aspectos fundamentales como, intersectorialidad, descentralización gestión sectorial local, participación y autorresponsabilidad de las comunidades. Lo anterior fundamenta el inicio del origen del APA (Atención Primaria Ambiental).

La Organización Panamericana de la Salud, OPS, en la Carta Panamericana sobre Ambiente y Salud en el Desarrollo Humano Sostenible, 1995, expresa lo siguiente.

«La participación de los individuos y las comunidades en mantener y mejorar sus ambientes de vida debe promoverse y apoyarse. La participación comunitaria debe plasmarse en estrategias para el desarrollo sustentable, incluyendo la Atención Primaria Ambiental, la Atención Primaria de Salud y la Educación de los niños y adultos. En cada nivel de la organización social y política se debe estimular y apoyar redes de intereses y personas que actúen en colaboración, a fin de fomentar la integración de preocupaciones y recursos sectoriales en procesos de desarrollo.»

Queda explicito como la salud y el ambiente van juntos para el desarrollo y bienestar de la humanidad propiciando mejoras en la calidad de vida, donde la responsabilidad del ecosistema es compartida por las partes involucradas, todas orientadas hacia el bien común.

La OPS (1997) expresa que la Atención Primaria Ambiental es una estrategia de la acción ambiental, básicamente preventiva y participativa, que reconoce el derecho del ser humano a vivir en un ambiente saludable y agradable y a informarse acerca de los riegos ambientales sobre la salud, el bienestar y la supervivencia.

En una experiencia práctica la OPS (2017), propició un Concurso de Experiencias Significativas en Promoción de la Salud en la Región de las Américas 2017, siendo Cuba la beneficiaria de la implementación de un proyecto de gestión participativa comunitaria en la Atención Primaria Ambiental, que permitió la incidencia política y abogacía, proponer, asesorar, monitorear y evaluar las políticas públicas, para contribuir a incrementar la salud de la población con enfoque de determinantes sociales.

El modelo de la APA busca reconocer como un espacio importante lo local, donde lo cotidiano cobre valor porque es allí donde las personas (niños (as), mujeres, hombres, jóvenes adultos, mayores) conviven con sus realidades y conocen el porqué de ellas.

Es este caso, es la comunidad Shuar de Naranjal, la que debe propulsar la participación de sus habitantes, en un proceso de dar soluciones a los problemas planteados se requiere la puesta en práctica de algunas variables tales como, el conocimiento de esa realidad en que viven, pero debe ser, más que un conocimiento, un empoderamiento. Esto se puede lograr, entre muchas de sus

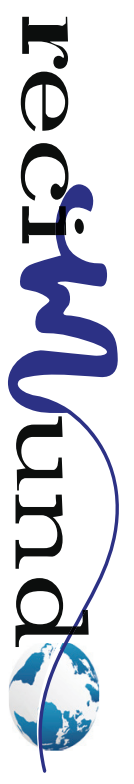


formas, a través de la participación comunitaria, el compromiso y la responsabilidad, lo que permite una participación en la toma de decisiones y el desarrollo de liderazgo, así se podrá tener comunidades activas que exigen y proponen cambios.

Según la OMS (1998), la meta es lograr la seguridad de la salud para todos y obtener equidad de la salud en todo el mundo. La APA recoge los propósitos de "salud para todos en el siglo XXI". La APA no es una negación ni una sustitución de la APS. Es una propuesta cualitativamente diferente, complementaria, que reconoce que los 20 años de APS han contribuido a una gran transformación social en todos los países, en lo que se refiere a descentralización y participación de la comunidad en la planificación, organización y funcionamiento de los sistemas de salud.

La estrategia de la APA se basa en los valores de equidad, participación, eficiencia e integración de la APS, pero va más allá al incluir la descentralización, el carácter interdisciplinario, la participación cívica, la organización, la prevención y protección del entorno, la diversidad, la cogestión y autogestión, la coordinación, la autonomía y la solidaridad.

Todo lo antes expuesto enlaza al Complejo turístico Shuar Tsuer Entsa el cual por su actividad termal requiere de una misión, una visión y unos objetivos orientados desde la filosofía de la Atención Primaria Ambiental, como norte de la calidad de sus servicios. Es fundamental la conservación del ambiente para que perdure el legado natural, humano y de los saberes ancestrales para que las futuras generaciones puedan disponer de estos espacios con una vision ecológica, ya que es esa riqueza vegetal un pulmón muy importante para el ejercicio de las prácticas de vida saludable que se desarrollan en este lugar.

\section{Materiales y métodos}

La metodología empleada para la Evaluación del Impacto Ambiental (EIA) es el Método de Leopold. Según Figueroa y col (1998), comenta que para el método cada impacto se lo considera en función de su magnitud e importancia. La magnitud de una interacción es su intención o escala y se describe mediante la designación de un valor numérico comprendido entre 1 y 10, donde 10 representa una gran magnitud. La importancia de una interacción está relacionada con lo significativa que esta sea o con la evaluación de las consecuencias probables del impacto previsto. Así mismo, la escala de importancia varía entre 1 y 10.

Según Allub (1990), esta matriz es un procedimiento estándar utilizado por el United States Geological Survey, creado por el Dr. Luna Leopold, para estimar impactos asociados con casi cualquier tipo de proyecto de construcción ". Su ventaja principal consiste en que incorpora información cualitativa de relaciones de causa - efecto y ayuda considerablemente a la exposición de los resultados de la investigación. 


\section{EL TURISMO EN SALUD Y ATENCIÓN PRIMARIAAMBIENTAL. CASO: COMPLEJO TURISTICO SHUAR TSUER ENTSA-BALAO CHICO ECUADOR}

Tabla 1. Tabla de leopold

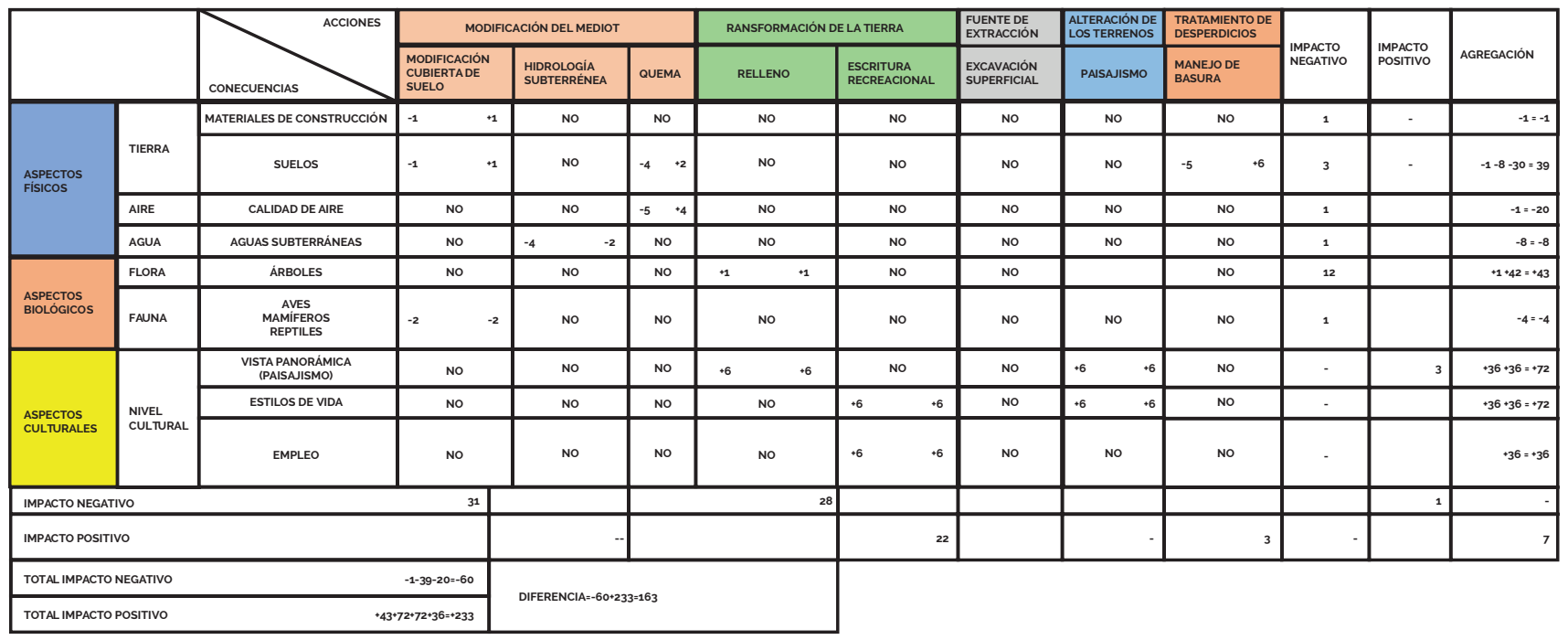

Fuente: Elaboración propia

\section{Discusión de resultados}

Los resultados obtenidos del EIA en el Complejo Turístico Shuar Tsuer Entsa para la identificación y evaluación de los impactos positivos y negativos arrojaron los siguientes resultados:

Impactos de carácter sanitario: Actualmente en la Comunidad Shuar de Naranjal se dan dos tipos:

Basura: La recolección de los desechos a nivel del complejo se hace de modo manual con una carretilla destinada para tales fines, los desechos están todos entremezclados, sin clasificación. El destino final se acumula en el fondo del estacionamiento y de ahí cuando pase el camión de la municipalidad. En la comunidad la recolección es irregular y algunos vecinos queman tales desechos, con la emanación de $\mathrm{CO}_{2}$ se contamina el aire.

Este impacto es negativo, directo, de magnitud e importancia media, permanente, localizado, reversible y recuperable.

En el estudio realizado por García et, al (2018), existe una gran producción de residuos reciclables que están siendo desapro- vechados y podrían representar una oportunidad de desarrollo económico, situación que se analiza en dos mercados de Machala, se considera importante el reciclaje y la clasificación de los desechos sólidos, lo cual coincide con lo sucedido en la comunidad al no hacer la selección de los desechos sólidos.

Servicios Sanitarios: En la comunidad ni en el Complejo existe sistema de recolección de aguas servidas, la misma se realiza a través del uso de pozos sépticos. Esto constituye un impacto negativo, de moderada magnitud e importancia, la contaminación del agua subterránea es una cuestión seria, en particular en las áreas donde los acuíferos proporcionan una gran parte del suministro de agua.

Un origen común de la contaminación del agua subterránea son las aguas fecales. Las bacterias peligrosas pueden ser filtradas mecánicamente por el sedimento a través del cual el agua percola, destruidas por oxidación química o asimiladas por otros microorganismos. Para que se produzca purificación, sin embargo, el acuífero debe ser de la composición correcta. Este aspecto amerita ser estudiado con mayor profundidad. 
Es así como Daus (2019), menciona que, la Asociación de Aguas y Aguas Residuales del Caribe (CWWA, por sus siglas en inglés), se ha destacado la importancia de preservar y restaurar los acuíferos subterráneos y se ha identificado como una intervención prioritaria del Caribe. En el caso del complejo turístico es necesario la preservación de estas aguas subterráneas, ya que constituyen parte importante del atractivo turístico.

Impactos sobre el suelo: La construcción de la infraestructura turística ha conducido a procesos de excavación superficial, ejecución de cortes, rellenos, limpieza y desbroce, lo cual marca en el suelo un impacto negativo, directo de moderada magnitud e importancia, temporal, localizado y recuperable. Al respecto el Colegio de Ingenieros de Mexico (2018), comentan que, los materiales utilizados en la construcción, muchas veces son elaborados con agentes tóxicos que contaminan la capa de ozono y dañan el aire. Además, la fabricación de éstos, implica que se incremente el agotamiento de recursos renovables y no renovables a causa de la extracción ilimitada de materias primas y del consumo de recursos fósiles.

\section{Impactos sobre la vegetación existente:}

Las actividades desarrolladas en la fase de construcción causan un impacto negativo sobre la vegetación de este bosque tropical, directo de moderada magnitud e importancia, temporal, localizado y recuperable. Se requiere la colocación de barreras naturales alrededor de las piscinas para evitar que las mismas se llenen de tierra, con lo cual se mejora la calidad visual del entorno, generando un impacto positivo de alta magnitud e importancia. Por su parte Montero (2020), comenta que, el componente más afectado es la alteración del suelo, debido a la remoción de la cobertura vegetal, apertura de zanjas, creación de vías de acceso y construcción de la planta. Finalmente, propone medidas correctivas en el plan de manejo ambiental para mitigar, prevenir y monitorear los impactos negativos que se generan.

Impactos sobre la fauna: Las actividades desarrolladas en la fase de construcción causan un impacto negativo sobre la fauna silvestre de este bosque tropical, directo de moderada magnitud e importancia, temporal, localizado y recuperable. Ahora con la nueva promoción de actividades de senderismo el impacto negativo se incrementa, porque el tránsito para llegar a las cascadas no es muy frecuentado.

En este orden de ideas, Borja (2019), refiere que los humanos pueden influir negativamente sobre la fauna silvestre, siendo los responsables de la destrucción del hábitat de las especies, así como de su fragmentación, al construir grandes infraestructuras. Estas y muchas otras modificaciones del medio, junto con la presencia del ser humano realizando actividades de ocio en la naturaleza, influyen en los animales silvestres provocándoles estrés fisiológico, repuesta común en todos los vertebrados.

Impactos sobre el recurso hídrico: Las aguas subterráneas son un recurso insustituible en buena parte del planeta, e imprescindible para la salud y para la buena marcha de la economía, el aprovechamiento tiene un impacto potencial negativo, ya que se está disminuyendo la reserva del recurso y a esto se suma la contaminación de los acuíferos, la falta de un manejo adecuado a la capacidad de sobrecarga podría afectar su caudal.

En concordancia con lo antes expuesto, Menchaca (2017), comenta que, el agua es el recurso natural que está asociado a la vida. Su disponibilidad en cuanto a cantidad y calidad establece los niveles de bienestar humano y el desarrollo económico de los países del mundo. Desafortunadamente la disponibilidad del agua está disminuyendo debido a múltiples factores sociales y económicos, lo que obliga a analizar los 
procesos que se desarrollan en la relación del hombre con el medio ambiente.

Impactos sobre el paisaje: La colocación de señaléticas y reforestación de algunas zonas del espacio geográfico produce un impacto positivo de alta magnitud e importancia.

\section{Impactos sobre los aspectos culturales:} Este es un impacto totalmente positivo de alta magnitud e importancia y permanente, que involucra a la comunidad, donde se considera importante la capacitación del personal para que la prestación de servicios sea de óptima calidad, explicarles los beneficios económicos y sociales por una administración adecuada de las bellezas naturales del espacio donde habitan.

Según los resultados de la matriz de Leopold las unidades de impacto positivo dan como resultado 223, frente a las unidades de impacto ambiental negativas 60 , dando una diferencia entre estos impactos de 163 unidades de impacto ambiental positivas, siendo los impactos positivos mayores que los negativos, estos últimos son mitigables y temporales.

\section{Conclusiones}

El termalismo, es un atractivo inigualable que convierte a las ciudades que tienen la fortuna de poseer aguas termales en destinos obligados de convocatoria local o mundial. Es importante considerar que como producto de la intervención humana se generan impactos ambientales, que en el corto plazo reducen su capacidad de proveer bienes y servicios derivados de la producción de recursos naturales, si no se establecen las medidas necesarias para tales fines.

En el Complejo Turístico Shuar Tsuer Entsa, el Estudio de Impacto Ambiental arrojo un número mayor de unidades de impacto ambiental positivas, siendo las unidades de impacto negativo mitigables y temporales.
A través de la Atención Primaria Ambiental se promueve la participación de sus habitantes, en un proceso de dar soluciones a los problemas ambientales encontrados, para ello se requiere la puesta en práctica de algunas variables tales como, el conocimiento de esa realidad en que viven, la capacitación del talento humano de la comunidad, pero debe ser, más que un conocimiento, un empoderamiento para hacer del Complejo un desarrollo sostenible y que sea el legado para las futuras generaciones.

\section{Bibliografía}

Allub, L. (1990). Impactos sociales de las grandes obras públicas: diagnóstico, evaluación y gestión. Universidad de Texas

Brandon, K (1993). Basic Steps Toward Encouraging Local Participation in Nature Tourism Projects. En: Ecotourism: A Guide for Planners and Managers. pp. 134-151. Ecotourism International Society. USA.

Borja, I (2019). Las actividades humanas estresan a los animales silvestres. Naturaleza (Tema) revista No 101.Disponible en: https://www.ecologistasenaccion.org/128487/las-actividades-humanas-estresan-a-los-animales-silvestres/

Calderón, G. (2016) Nuevo termalismo. Diario EL TELÉGRAFO: tomado de http://www.eltelegrafo. com.ec/noticias/columnistas/1/nuevo-termalismo

Correa, J. (2017) América Latina: Los destinos más demandados por el turismo de salud Tomado de: http:// www. clustersalud.americaeconomia.com/ america-latina-los-destinos-mas-demandados-turis. Recuperado 14/05/2017

Colegio Mexicano de Ingenieros Civiles (2018). Impacto ambiental durante el proceso de construcción. Disponible en: https://cmicac. com/2018/12/13/impacto-ambiental-durante-el-proceso-de-construccion/

Daus, A (2019). Almacenamiento y recuperación de Agua en Acuíferos. Banco Interamericano de Desarrollo. Disponible en: https://publications. iadb.org/publications/spanish/document/Almacenamiento_y_recuperaci\%C3\%B3n_de_agua_en_ acu\%C3\%ADferos_Mejoramiento_de_la_seguridad_en_el_abastecimiento_de_agua_en_el_Caribe._Oportunidades_y_desaf\%C3\%ADos_es.pdf

Fayos Solá, E (1997). Educación y Formación Turística: la visión de la OMT. En: El Capital Humano en la Industria Turística del siglo XXI. OMT, Madrid, 1997. 
Figueroa, A y col (1998). Evaluación de impacto ambiental: un instrumento para el desarrollo. Ed. Centro de Estudios Ambientales para el Desarrollo Regional, Bogotá

Fuentes, R. (1995) Análisis de las principales características de la demanda de Turismo Rural en España. En: Revista Estudios Turísticos № 127. pp. 19-52. Madrid, España.

García Batista, Rigoberto Miguel, Socorro Castro, Alejandro Rafael, \& Vanessa Maldonado, Ana. (2019). Manejo y gestión ambiental de los desechos sólidos, estudio de casos. Revista Universidad y Sociedad, 11(1), 265-271. Epub 02 de marzo de 2019. Recuperado en 16 de agosto de 2021, de http://scielo.sld.cu/scielo.php?script=sci_arttext\&pid=S2218-36202019000100265\&In$\mathrm{g}=$ es\&tlng=es.

Gómez, V. (2019). Matriz de Leopold: para qué sirve, ventajas, ejemplos. Lifeder. Recuperado de https://www.lifeder.com/matriz-de-leopold/.

Loor, L; Alonso, A \& Pérez, M. (2017). La actividad turística en el Ecuador: ¿Turismo Consciente o Turismo Tradicional? Revista ECA Sinergia. Facultad de Ciencias Administrativas y Económicas. U.T.M. junio 2018 Vol. 9 № 1 pp 97-108 Manabí, Ecuador.

Martínez, L. (1998) Turismo Rural "Una alternativa de agronegocios". En: Turismo Rural y Desarrollo Sustentable. Synergy Consultores Ltda. pp. 17-41. Santiago.

Menchaca, S (2017). Actividades humanas y el agua. Diario Xalapa Cultura. Disponible en: https:// www.uv.mx/cienciauv/files/2017/09/030-CYL-ACTIVIDADES-HUMANAS-Y-EL-AGUA-01.pdf

Ministerio De Turismo Ecuador (2012) Análisis del Sector Turismo. PROECUADOR

Ministerio de Comercio y Turismo (1994). Manual del Planificador de Turismo Rural. Madrid, España

Montero-Vega, F. S., Molina-Cedeño, C. S., Pillco-Herrera, B. M., Sarduy-Pereira, L. B., \& Diéguez-Santana, K. (2020). Evaluación del impacto ambiental de la construcción de una planta de tratamiento de aguas residuales. Caso río Pindo Chico, Puyo, Pastaza, Ecuador. Ciencia, Ambiente y Clima, 3(1), 23-39. Doi: https://doi.org/10.22206/ cac.2020.v3i1.pp23-39

Mourelle, M. (2009) Técnicas hidrotermales y estética del bienestar e. Nobel SA. Madrid

Organización Mundial de la Salud (OMS, 1998). La salud para todos en el siglo XXI. $51^{\text {a }}$ Asamblea Mundial de la Salud, tomada de: www.who.int , WHO IRIS , Headquarters , Governing bodies , WHA51
Organización De Las Naciones Unidas Para La Educación, La Ciencia Y La Cultura (UNESCO, 1978) Islas Galápagos tomadas de: http //www. whc. unesco.org/es/list/1

Organización Panamericana de la Salud (OPS, 1995). Salud y ambiente en el Desarrollo Humano Sostenible de las Américas. Publicaciones Cepis

Organización Panamericana de la Salud (OPS, 1997). Atención Primaria Ambiental. Washington, DC. (Documento interno).

Organizacion Panamericana de la Salud (OPS,2017). Concurso de Experiencias Significativas en Promoción de la Salud en la Región de las Américas 2017

Rivas, H (1996). Hacia un desarrollo turístico de los ambientes naturales en Chile. Revista Ambiente y Desarrollo

Rivas, A (2020). Importancia económica, cultural, social y turística de las termas en la provincia de Pichincha. Rev. Digital Publisher V5-N3 (may-jun) 2020, pp. 133-153

Santos, G. (2016) Turismo de bienestar genera US\$ 26.000 millones en Latinoamérica. www. hosteltur.lat/124000_turismo-bienestar-genera-us-26000-millones-latinoamerica.html

Secall, R; Torres, E; Fuentes R y Martin, M (2006) Estructura de mercados turísticos Ed UOC, Barcelona.Pg. 174

Silega, G. (2010) Tratado de Medicina Física Hidrología y Climatología Médica. Ed. Hipócrates Production USA Pg. 14

Suarez, M. (2016) Latinoamérica: ¿Crisis o Marketing Termal?, tomado de http://www.termasworld. com

Toledo Rodríguez, Odalys del Carmen, \& de Aparicio, Xiomara. (2018). El desarrollo sostenible mediante el turismo de salud termal en el complejo turístico Shuar Tsuer Entsa. Revista Universidad y Sociedad, 10(2), 61-66. Epub 03 de febrero de 2018. Recuperado en 13 de julio de 2021, de http://scielo.sld.cu/scielo.php?script=sci_arttext\&pid=S2218-36202018000200061\&lng=es\&tln$g=e s$.

Wallingre, N. (Comp.) (2018). Desarrollo del turismo en América Latina: fases, enfoques e internacionalización. Bernal, Argentina: Universidad Nacional de Quilmes, Unidad de Publicaciones del Departamento de Economía y Administración. Disponible en RIDAA-UNQ Repositorio Institucional Digital de Acceso Abierto de la Universidad Nacional de Quilmes http://ridaa.unq.edu.ar/hand- 


\section{le/20.500.11807/1022}

World Tourism Organization (2015) Tourism Highlights ¿Qué países del mundo reciben más turistas? ¿Y en América Latina ...tomado de: www.aprendedeturismo.org/que-países-del-mundo-que-reciben-más-turistas-y-en-América

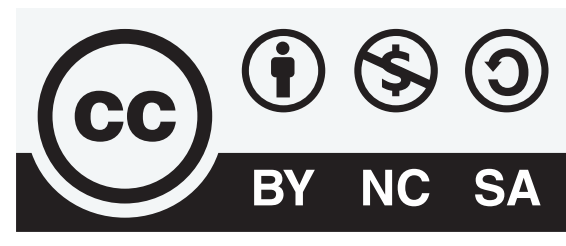

CREATIVE COMMONS RECONOCIMIENTO-NOCOMERCIAL-COMPARTIRIGUAL 4.0 .

\section{CITAR ESTE ARTICULO:}

Peraza de Aparicio, C. X. (2021). El turismo en salud y atención primaria ambiental. Caso: Complejo turístico Shuar Tsuer Entsa-Balao Chico Ecuador. RECIMUNDO, 5(1 (Suple), 44-55. https://doi.org/10.26820/recimundo/5.(Suple1). oct.2021.44-55 